\title{
Seebeck Coefficient and Optical Studies of Cadmium Doped $\mathrm{CuInS}_{2}$ Single Crystal
}

\author{
S.H. CHAKI* \\ Department of Physics, Sardar Patel University, Vallabh Vidyanagar - 388 120, Gujarat, India
}

(Received January 15, 2009; in final form April 16, 2009)

\begin{abstract}
Single crystals of $\mathrm{CuInS}_{2}$ were successfully grown by chemical vapor transport technique using iodine as transporting agent. The as-grown chemical vapor transport $\mathrm{CuInS}_{2}$ single crystals were found to have large resistivity. To decrease the resistivity of the crystals they were doped with three different cadmium concentrations. Seebeck coefficient variation with temperature is measured for all the three doped samples. Optical bandgaps were determined for these three doped samples using optical absorption spectra. The obtained results are discussed in detail.

PACS numbers: 71.20.Nr, 72.20.Pa, 78.20.-e, 61.50.-f
\end{abstract}

\section{Introduction}

The I-III- $\mathrm{VI}_{2}$ compounds are the ternary analogs of the binary II-VI compounds and have received much attention, as they can be made both $n$ - and $p$-type $[1,2]$.They crystallize in the chalcopyrite structure, which is closely related to that of the zinc-blende structure [3]. Amongst different chalcopyrite semiconducting materials, $\mathrm{CuInSe}_{2}$ and $\mathrm{CuInS}_{2}$ have received much attention because their band gaps match well with the solar spectrum leading to photovoltaic applications $[4,5]$. The most widely studied semiconductor of this group is $\mathrm{CuInSe}_{2}$, for its fundamental properties with a view to prepare devices $[6,7]$. Very high efficiencies have been reported with copper indium gallium selenide as the absorber layer [7]. The semiconductor containing selenium is harmful due to the toxicity of selenium. Therefore, $\mathrm{CuInS}_{2}$, an equally important but environmentally benign solar cell material has great importance in current photovoltaic research. An efficiency of $\approx 16 \%$ has already been exhibited in solar cells fabricated with $\mathrm{CuInS}_{2}$ thin films with an area of $1 \mathrm{~cm}^{2}[8]$. Apart from photovoltaic applications, the compound is also investigated for practical applications in non-linear optical devices [9-11]. In order to control a conduction type and obtain a low resistivity, several impurities have been studied [12-17]. For any applications the availability of single crystals of the compounds is highly desirable. Single crystal growth from melt, however, is hindered by a solid state phase transition at $990^{\circ} \mathrm{C}$ [18], at which the crystal passes from the zinc-blende structure with a disordered cation distri-

* e-mail: sunilchaki@yahoo.co.in bution to the ordered chalcopyrite structure upon cooling. The transition can give rise to a severe cracking of the prepared single crystals. The existence of a solid state transition is therefore an influential factor in choosing an appropriate crystal growth technique, e.g. growth from the melt, solution, or vapor. The author in this paper reports the growth of $\mathrm{CuInS}_{2}$ single crystals well below the phase transition temperature by employing chemical vapor transport (CVT) technique. It has been found that the group II ( $\mathrm{Zn}$ and $\mathrm{Cd}$ ) atoms, which are possible donors on the $\mathrm{Cu}$ site and acceptors on the group III site, act primarily as donors $[19,20]$. Thus the as-grown CVT single crystals were doped with three different concentrations of cadmium to reduce their resistivity. The Seebeck coefficient variation with temperature and ambient optical properties of these cadmium doped single crystals were studied.

\section{Experimental procedure \\ 2.1. Crystal growth and doping}

The single crystals of $\mathrm{CuInS}_{2}$ were grown by CVT technique using iodine as transporting agent. Pure elements of copper (Johnson-Mathey Chemicals Ltd., UK), indium and sulphur (Chiti-Chem Corp, Vadodara, India) of $5 \mathrm{~N}$ purity were taken in stoichiometric proportion and sealed in an evacuated $\left(\approx 10^{3} \mathrm{~Pa}\right)$ quartz ampoule for compound preparation. The synthesis of the compound was carried out by heating the ampoule gradually up to $1373 \mathrm{~K}$ and holding at that temperature for $12 \mathrm{~h}$.

After being slowly cooled down, the synthesized compound was homogenized by grinding with agate mortar and then transferred into an evacuated $\left(\approx 10^{3}\right)$ quartz growth ampoule containing $10 \mathrm{mg} / \mathrm{cc}$ iodine transporter. 
The sealed ampoule was then placed in a two-zone coaxial horizontal furnace with the reaction zone at a higher temperature and the growth zone at a lower temperature. The details of the growth run are tabulated in Table I.

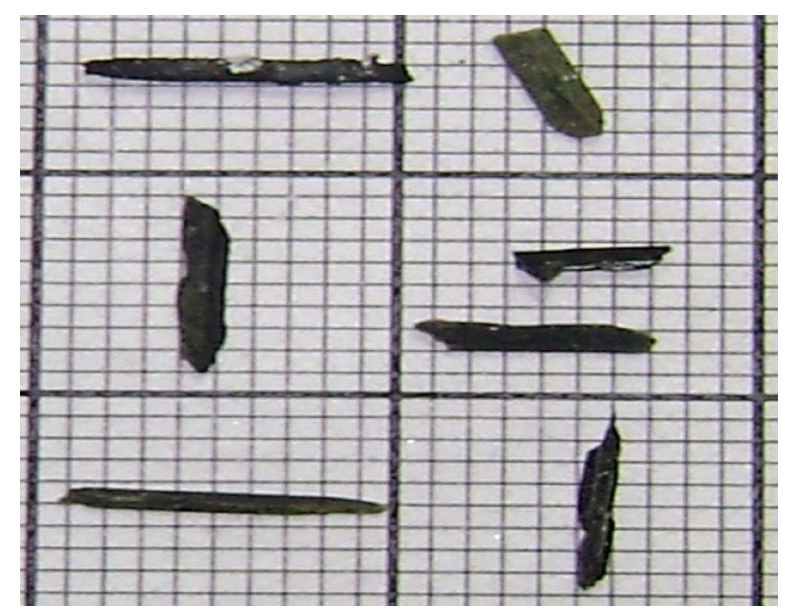

Fig. 1. Large size single crystals of $\mathrm{CuInS}_{2}$.

TABLE I

Growth parameters of $\mathrm{CuInS}_{2}$ single crystals.

\begin{tabular}{c|c|c|c|c|c}
\hline \hline \multicolumn{2}{c|}{$\begin{array}{c}\text { Compound } \\
\text { preparation }\end{array}$} & \multicolumn{4}{|c}{ Single crystals growth } \\
\cline { 3 - 4 } \multicolumn{2}{c}{} & \multicolumn{2}{c|}{$\begin{array}{c}\text { temperature } \\
\text { distribution }\end{array}$} & growth & ampoule \\
\hline $\begin{array}{c}\text { temperature } \\
{[\mathrm{K}]}\end{array}$ & $\begin{array}{c}\text { time } \\
{[\mathrm{h}]}\end{array}$ & $\begin{array}{c}\text { hot zone } \\
{[\mathrm{K}]}\end{array}$ & $\begin{array}{c}\text { cold zone } \\
{[\mathrm{K}]}\end{array}$ & $\begin{array}{c}\text { time } \\
{[\mathrm{h}]}\end{array}$ & $\begin{array}{c}\text { dimensions } \\
{[\mathrm{mm} \times \mathrm{mm}]}\end{array}$ \\
\hline 1373 & 12 & 1093 & 1023 & 144 & $14 \times 14(\mathrm{ID})$
\end{tabular}

The resulting single crystals were needle shaped and shining black in color, Fig. 1. The average dimensions of the large size single crystals thus grown were about $9 \times 1 \times 1 \mathrm{~mm}^{3}$.

\subsection{Characterization}

The stoichiometric composition of the as-grown single crystal was determined through energy dispersive analysis of X-rays (EDAX) coupled with electron microscopy (Philips EM-400). The EDAX data of weight $\%$ of the as-grown single crystals are copper $26.176 \%$, indium $48.869 \%$, and sulfur $24.955 \%$.

The crystallographic lattice parameters of the as-grown single crystals were determined using X-ray diffraction (XRD) (X-pert-MPD) employing $\mathrm{Cu} K_{\alpha}$ radiation, Fig. 2. All the peaks could be indexed as that of $\mathrm{CuInS}_{2}$ with tetragonal chalcopyrite structure. The deduced lattice parameters were $a=5.505 \AA$ and $c=11.324 \AA$.

The as-grown crystals were found to be $n$-type and semi-insulating, with room temperature resistivity of the order of $10^{5}-10^{6} \Omega \mathrm{cm}$. To make the crystals suitable for any application, the resistivity value has to be reduced. The resistivity value was decreased by doping

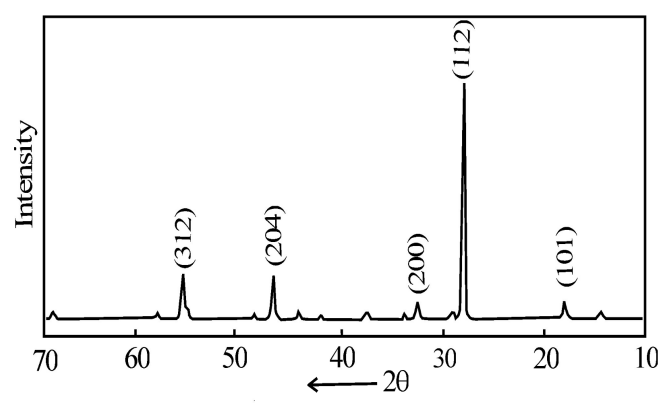

Fig. 2. X-ray diffraction pattern of $\mathrm{CuInS}_{2}$.

the crystals with dopant. Cadmium was found to be a suitable dopant. Three different doping concentrations were tried. The dopant and the grown single crystals were sealed in an evacuated $\left(\approx 10^{-3} \mathrm{~Pa}\right)$ quartz ampoule and held at annealing temperature for definite time period. The different doping concentration and annealing conditions employed are tabulated in Table II.

TABLE II

Annealing parameters.

\begin{tabular}{c|c|c|c}
\hline \hline $\begin{array}{c}\text { Sample } \\
\text { No. }\end{array}$ & $\begin{array}{c}\text { Cadmium } \\
\text { concentration }[\mathrm{mg} / \mathrm{cc}]\end{array}$ & $\begin{array}{c}\text { Annealing } \\
\text { temperature }[\mathrm{K}]\end{array}$ & $\begin{array}{c}\text { Annealing } \\
\text { time }[\mathrm{h}]\end{array}$ \\
\hline S1 & 1.00 & 973 & 10 \\
S2 & 0.50 & 973 & 10 \\
S3 & 0.25 & 973 & 10
\end{tabular}

After annealing, the samples were cleaned in concentrated $\mathrm{HCl}$ acid to remove any surface deposit of cadmium.

Thermoelectric power measurements on the three cadmium doped $\mathrm{CuInS}_{2}$ samples (samples S1, S2, and S3) were made using Differential Temperature Controller fabricated by Scientific Solutions, Mumbai. With the setup, it is possible to measure emf in temperature from ambient to $423 \mathrm{~K}$ and $\Delta T$ to $\pm 10 \mathrm{~K}$ simultaneously with better than $\pm 1 \mathrm{~K}$ stability. The problem usually encountered in making thermoelectric power measurements are stray thermal emfs. In the present setup, this has been eliminated by providing choice of selection of temperature gradient in the range 1 to $10 \mathrm{~K}$. Thermoelectric power measurement could not be accomplished on the as-grown single crystals of $\mathrm{CuInS}_{2}$, because of its very high resistivity.

The optical absorption spectra on cadmium doped $\mathrm{CuInS}_{2}$ single crystal samples (samples S1, S2 and S3) were obtained in an UV-VIS-NIR Shimadzu Spectrophotometer UV-365 in the wavelength range $400 \mathrm{~nm}$ to $900 \mathrm{~nm}$ at room temperature. For these measurements, thin crystals of thickness about $0.3 \mathrm{~mm}$ were used. The samples were pasted on a thick black paper with a cut exposing the crystals to the incident radiation. The reference used was a replica of the black paper having the cut in exactly the same position. 


\section{Results and discussions}

Single crystals of $\mathrm{CuInS}_{2}$ of fairly large size have been successfully grown by a CVT technique using iodine as transporter. The chemical composition of the as-grown single crystals using EDAX showed that the crystals possess desired stoichiometry. The crystallographic lattice parameters of the as-grown single crystals were determined using X-ray diffraction, Figure 2. All the peaks could be indexed as that of $\mathrm{CuInS}_{2}$ on a tetragonal crystal structure (JCPDS card No. 27-015) with space group of $I \overline{4} 2 d$. The deduced lattice parameters were in good agreement with the reported values [21].

The thermoelectric power $S$ variation as a function of the reciprocal of temperature $\left(T^{-1}\right)$ for all the cadmium doped samples S1, S2 and S3 is shown in Fig. 3. The absolute value of thermoelectric power is negative over the entire temperature range and therefore electrons are the dominant charge carriers for all the three doped samples stating them to be $n$-type semiconductor. The Seebeck coefficient $S$ for an $n$-type semiconductor can be expressed as [22]:

$$
S=-\frac{k}{e}\left(A+\frac{E_{\mathrm{F}}}{k T}\right) \text {, }
$$

where $k$ is the Boltzmann constant, $e$ is the electronic charge, $E_{\mathrm{F}}$ is the separation of the Fermi level from the bottom of the conduction band and $A$ is the scattering coefficient. This equation suggests that if $S$ is plotted against the reciprocal of temperature $\left(T^{-1}\right)$ a straight line should result. From the slope of this line, the value of $E_{\mathrm{F}}$ can be determined. The variation of $S$ with reciprocal of temperature $\left(T^{-1}\right)$ for the samples $\mathrm{S} 1, \mathrm{~S} 2$ and $\mathrm{S} 3$, Figure 3 shows a straight line in perfect conjecture with Eq. (1). The values of $E_{\mathrm{F}}$ calculated from the plots are represented in Table III.

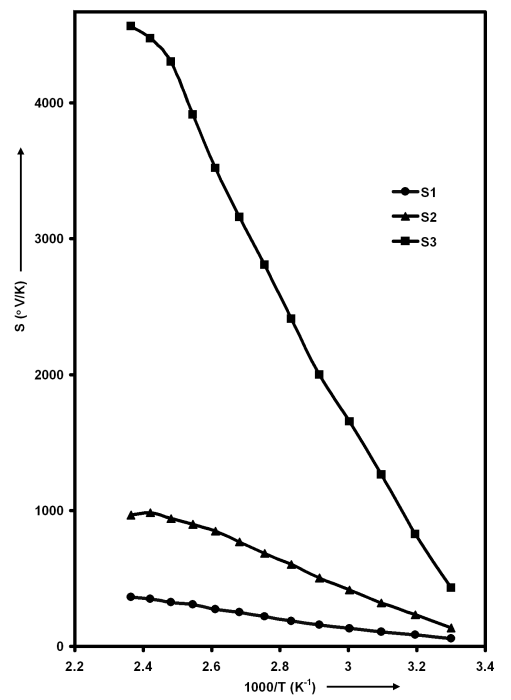

Fig. 3. Variation of thermoelectric power $S$ with reciprocal of temperature of the S1, S2, and S3 samples of cadmium doped $\mathrm{CuInS}_{2}$.
TABLE III

Room temperature Fermi energy, Seebeck coefficient and optical bandgap for $n$-type samples.

\begin{tabular}{c|c|c|c}
\hline \hline $\begin{array}{c}\text { Sample } \\
\text { No. }\end{array}$ & $\begin{array}{c}\text { Fermi energy } \\
E_{\mathrm{F}}[\mathrm{eV}]\end{array}$ & $\begin{array}{c}\text { Seebeck coefficient } \\
{[\mu \mathrm{V} / \mathrm{K}]}\end{array}$ & $\begin{array}{c}\text { Optical bandgap } \\
E_{\mathrm{g}}[\mathrm{eV}]\end{array}$ \\
\hline S1 & 0.017 & -57.0 & 1.36 \\
S2 & 0.041 & -136.0 & 1.40 \\
S3 & 0.13 & -430.0 & 1.44
\end{tabular}

Convers Herring [23] explained thermoelectric power behavior for impurity semiconductors by categorizing impurity concentrations into three groups. When the impurity concentration is very low, nearly all the charge carriers move as isolated entities in the regions of perfect crystalline material, and their motion can be rigorously described in terms of the concepts of band theory, at least if the mobility is high enough to justify treating the interaction with the lattice vibrations as a small perturbation on the motion of the carriers. This illustrates non-degenerate semiconducting behavior. In the present study, sample S3 having low cadmium doping concentration behaves as a non-degenerate semiconductor. It is substantiated by the highest separation of Fermi level from the bottom of the conduction band and the thermoelectric power variation with temperature is steep, indicating a characteristic non-degenerate semiconducting behavior.

As the impurity concentration increases further, it becomes necessary to take into account the impurity scattering and related effects. The jumping of charge carriers from one impurity atom to the other forms an impurity band conduction which mingles and competes with normal conduction [24-26]. The onset of the Fermi degeneracy normally occurs in this impurity range. Thus sample S2 having cadmium doping concentration higher than S3 but lower than S1, can be considered as degenerate semiconductor having the Fermi level nearer to the conduction band in comparison to S3. The thermoelectric power variation with temperature is less steep than that of sample S3.

When the impurity concentrations get very high, the carriers can be treated as a degenerate Fermi gas and the impurities as mere sources of scattering. The sample shows metallic behavior. Here, sample S1 having highest cadmium doping concentration ought to behave as metal. The metallic behavior of S1 is being corroborated by its closest positioning of the Fermi level from the conduction band and the variation of thermoelectric power with temperature is least.

Moreover, it has been observed that with the increase in cadmium content in the cadmium doped $\mathrm{CuInS}_{2}$, the values of room temperature Seebeck coefficient become less negative (Table III), showing a tendency towards the metallic side in good agreement with reported behavior [27].

The optical absorption data was analyzed in terms of the theory of Bardeen et al. [28], which gives for a direct transition 


$$
\alpha=\frac{\beta\left(h \nu-E_{\mathrm{g}}\right)^{\gamma}}{h \nu},
$$

where $\beta$ is a constant, $E_{\mathrm{g}}$ is the optical bandgap and $h \nu$ is the energy of the incident photon, $\gamma$ assumes values $1 / 2$ and 2 for allowed direct and indirect transitions respectively. It is observed that for cadmium doped samples, the absorption can be satisfactorily explained with $\gamma=1 / 2$ in the energy range $400 \mathrm{~nm}$ to $900 \mathrm{~nm}$. The plot of $(\alpha h \nu)^{2}$ versus $h \nu$ for different samples S1, S2 and S3 is shown in Fig. 4. Ideally the graph should be linear, the deviation from linearity at lower incident photon energies can be attributed to the presence of structural irregularities and imperfections in these crystals.

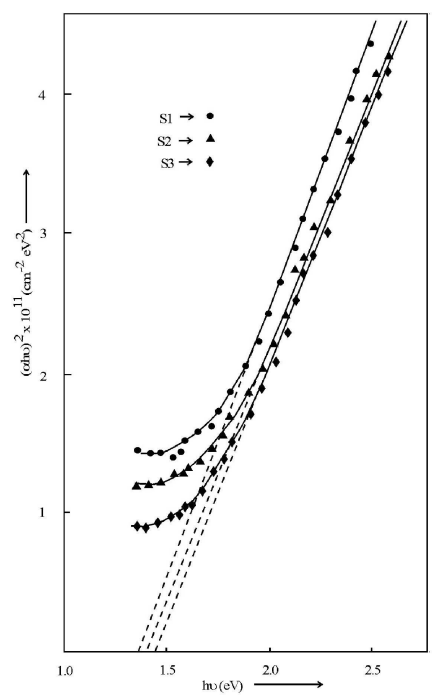

Fig. 4. Variation of optical absorption $(\alpha h \nu)^{2}$ as a function of photon energy $h \nu$ of the S1, S2, and S3 samples of cadmium doped $\mathrm{CuInS}_{2}$.

The intercept obtained by extrapolation of the linear portion of the plot on the energy axis gives a bandgap value for all the three samples. From the functional dependence obtained for the absorption coefficient on photon energy, it may be seen that the transition is a direct allowed one. The values of optical bandgap $E_{\mathrm{g}}$ obtained from the plots are given in Table III.

The bandgap values show that the optical energy bandgap decreases with increase in cadmium concentration. This can be attributed to the presence of shallow donor state in the cadmium doped $\mathrm{CuInS}_{2}$.

\section{Conclusions}

1. Average size good quality single crystals of $\mathrm{CuInS}_{2}$ have been successfully grown by CVT technique using iodine as transporting agent. The as-grown crystals have been characterized by EDAX and XRD techniques. The as-grown $\mathrm{CuInS}_{2}$ single crystals were doped with three different concentrations of cadmium.
2. The $n$-type nature of conduction was confirmed by the negative sign of absolute values of Seebeck coefficient $S$ for all the three doped samples. The variation of Seebeck coefficient $S$ with reciprocal temperature shows that the magnitude of variation with temperature is different in all the three doped samples. The highest doped sample S1 shows metallic behavior, sample S2 having intermediate doping concentration shows degenerate semiconducting behavior and the least doped sample S3 shows non-degenerate semiconducting behavior.

3. From the analysis of the optical absorption, it can be concluded that the energy bandgap decreases with the increase in the cadmium content.

\section{References}

[1] B. Tell, J.L. Shay, H.M. Kasper, J. Appl. Phys. 43, 2469 (1972).

[2] H.J. Hsu, N.H. Yang, R.S. Fang, I.M. Hsu, H.L. Hwang, J. Cryst. Growth 20, 83 (1984).

[3] J.J.M. Binsma, L.J. Giling, J. Bloem, Phys. Status Solidi A 63, 595 (1981).

[4] M.A. Green, K. Emery, D.L. King, S. Igari, W. Warta, Prog. Photovolt. Res. Appl. 10, 355 (2002).

[5] D. Cahen, G. Dagan, Y. Mitrovsky, G. Hodes, W. Giriat, M. Lubke, J. Electrochem. Soc. 132, 1062 (1985).

[6] J. Hedstrom, H. Ohlsen, M. Bodegard, A. Kyler, L. Stolt, D. Hariskos, M. Rukch, H.W. Schock, in: Proc. 23rd IEEE Photovoltaic Specialist Conf., Louisville, IEEE, New York 1993, p. 364.

[7] M.A. Contreras, A.M. Gabor, A.L. Tennant, A. Asher, J.R. Tuttle, R. Noufi, Prog. Photovolt. 2, 287 (1994).

[8] H.W. Schock, in: Proc. 12th European Photovoltaic Solar Energy Conf., 1994, Eds. R. Hill, W. Palz, P. Helm, Stephens, Bedford (UK) 1994, p. 944.

[9] S.D. Mittleman, R. Singh, Solid State Commun. 22, 155 (1977).

[10] S. Mora, N. Romeo, L. Tarricone, Solid State Commun. 29, 155 (1979).

[11] H. Neumann, W. Horig, V. Savelev, J. Lagzdonis, B. Schumann, G. Kuhn, Thin Solid Films 79, 167 (1981).

[12] M. Ben Rabeh, M. Kanzari, B. Rezig, Thin Solid Films 515, 5943 (2007).

[13] Y. Akaki, H. Matsuo, K. Yoshino, Phys. Status Solidi C 8, 2597 (2006).

[14] M. Abaab, M. Kanzari, B. Rezig, M. Brunel, Sol. Energy Mat. Sol. Cells 59, 299 (1999).

[15] G. Brandt, A. Ranber, J. Schneider, Solid State Commun. 12, 481 (1983).

[16] J.J.M. Binsma, L.J. Giling, J. Bloem, J. Lumin. 27, 35 (1982).

[17] H.Y. Unegt, H.L. Hwang, J. Phys. Chem. Solids 51, 11 (1990). 
[18] J.J.M. Binsma, L.J. Giling, J. Bloem, J. Cryst. Growth 50, 429 (1980).

[19] T. Yamamoto, H.K. Yoshida, Jpn. J. Appl. Phys. 35, L1562 (1996).

[20] J.L. Shay, J.H. Wernick, Ternary Chalcopyrite Semiconductors: Growth, Electronic Properties, and Applications, Pergamon Press, Oxford 1971, p. 188.

[21] N.A. Goryunova, The Chemistry of Diamond like Semiconductor, MIT, Cambridge (MA) 1965, p. 142.

[22] H.B. Kwok, H.R. Bube, J. Appl. Phys. 44, 138 (1973).
[23] Convers Herring, Phys. Rev. 96, 1163 (1954).

[24] K. Shifrin, J. Phys. (USSR) 8, 242 (1944).

[25] C. Erginsoy, Phys. Rev. 80, 1104 (1950).

[26] C. Erginsoy, Phys. Rev. 88, 893 (1952).

[27] R.W. Fritts, in: Materials Technology Series - Thermoelectric Materials and Devices, Eds. I.B. Cadoff, E. Miller, Chapman \& Hall, London 1960, p. 152.

[28] J. Bardeen, F.J. Blatt, L.H. Hall, in: Photoconductivity Conference, Eds. R. Breckenridge, B. Russell, E. Hahn, Wiley, New York 1956, p. 146. 